\title{
Sobre el estilo: Susan Sontag, Marta Traba y Leyla Perrone-Moisés \\ About Style: Susan Sontag, Marta Traba and Leyla Perrone-Moisés
}

\author{
Hugo Herrera Pardo \\ Pontificia Universidad Católica de Valparaíso \\ hugo.herrerap@gmail.com
}

\section{Resumen}

Una serie de rasgos en común permiten pensar de manera relacionada ciertas zonas de las obras desarrolladas por la norteamericana Susan Sontag, la argentino-colombiana Marta Traba y la brasileña Leyla Perrone-Moisés. Algunos de estos rasgos son su contemporaneidad, su dedicación al género ensayístico, que la década del sesenta fuera un periodo importante dentro de sus respectivas bibliografías y que en los libros publicados por ellas en aquel decenio se decantara una preocupación compartida por reflexionar sobre la categoría de estilo. El presente artículo propone una reflexión, comparada y contrastiva, en torno a los puntos de contacto y de diferencia que sobre tal noción expusiera Susan Sontag en su ensayo "Sobre el estilo" (incluido en su célebre libro Contra la interpretación y otros ensayos, 1966), Marta Traba en su importante texto Los cuatro monstruos cardinales (1965) y Leyla Perrone-Moisés en los textos integrantes de su primer libro O nôvo romance francês (1966) y también en escritos adyacentes a aquella publicación.

Palabras clave: Susan Sontag, Marta Traba, Leyla Perrone-Moisés, ensayo, estilo.

\section{Abstract}

A series of features in common allow us to think about certain areas of the works developed by the American Susan Sontag, the Argentinian-Colombian Marta Traba and the Brazilian Leyla Perrone-Moisés. Some of these features are their contemporaneity, their dedication to the essayist genre, that the sixties were an important period within their respective bibliographies and that in the books published by them in that decade there is a shared concern to reflect on the category of style. This article proposes a reflection, compared and contrastive, about the points of contact and difference that Susan Sontag presented in this notion in her essay "On style" (included in her famous book Against Interpretation and Other Essays, 1966 ), Marta Traba in her important text The Four Cardinal Monsters (1965) and Leyla Perrone-Moisés in the texts of her first book O nôvo romance francês (1966) and also in writings adjacent to that publication.

Keywords: Susan Sontag, Marta Traba, Leyla Perrone-Moisés, essays, style. 


\section{Devenires de "lo nuevo"}

Sobre la relación entre escritura y percepción de "lo nuevo" (sea esta valoración positiva o no) podría fundarse una ontología de la cultura, entendiendo por esta a un inevitable trabajo de inclusión y exclusión, al modo en que fue pensado, por ejemplo, por Maurice Blanchot. Esa "potente maquinaria colectiva que silenciosa e insensiblemente, día y noche, prosigue su tarea" (59) es como el pensador francés metaforizó a los "grandes reductores" que operan (operamos) en el ámbito de la cultura. Podríamos decir que es a partir de las posiciones que adoptamos ante o contra "lo nuevo" — definido ya sea por un criterio de actualidad o de retorno de lo anteriormente excluido- que, por lo general, los actores de un campo cultural irrumpen en él o definen sus posiciones. Pensemos en una ensayista como Susan Sontag y en el epílogo que escribió, treinta años después, ${ }^{1}$ a su primera colección de ensayos Contra la interpretación y otros ensayos (2008 [1966]). Allí retrospectivamente Sontag señala que las “jerarquías (alto/bajo) y las polaridades (forma/contenido, intelecto/sentimiento) que ponían en entredicho inhibían la correcta comprensión de la obra nueva que admiraba. Aunque no asumía un compromiso de programa con lo «moderno», adoptar la causa de la obra nueva, sobre todo la que había sido desdeñada o ignorada o incomprendida, parecía más útil que escribir sobre viejas predicciones" (390). Me interesa reflexionar en las siguientes páginas sobre este problema entre escritura ensayística y percepción de "lo nuevo", ubicando en paralelo el posicionamiento que Susan Sontag adoptó en su primer libro de ensayos con otros dos planteamientos concomitantes en varios sentidos (que serán argumentados posteriormente); el que desplegara la crítica de arte argentino-colombiana Marta Traba en su libro Los cuatro monstruos cardinales (1965) y la desarrollada por la ensayista brasileña Leyla Perrone-Moisés en algunos de sus textos publicados durante la década de los sesenta, entre ellos su primera colección de ensayos O nôvo romance francês (1966).

No obstante, antes de avanzar hacia aquella argumentación se torna necesario reconocer como condición de la misma un debate contemporáneo sobre la relación entre modernidad y "lo nuevo". Y es que una de las hipótesis que recientemente ha atravesado con mayor potencia campos como la filosofía política o la crítica cultural ha sido aquella que ha establecido vínculos entre la fase contemporánea del capitalismo y la percepción temporal centrada en lo retrospectivo, a partir de sus efectos en la vida cotidiana y en la producción de cultura. Referida en expresiones o conceptos tales como "la lenta cancelación del futuro", a decir de Franco "Bifo" Berardi, "anacronismo posmoderno", a juicio de Mark Fisher o "retromanía”, según la noción de Simon Reynolds, esta hipótesis atiende a cierta dislocación temporal, a cierta discronía que puede percibirse como un concentrado repliegue de la temporalidad que posibilita,

1 Dicho texto fue escrito por Susan Sontag para la traducción al castellano de Against Interpretation and Other Essays que Alfaguara publicó en 1996. Con posterioridad, y previa revisión, fue incluido en Cuestión de énfasis (2007). 
de este modo, la sensación de una compleja simultaneidad por sobre el desarrollo lineal previamente dominante. En Después del futuro. Desde el futurismo al cyberpunk. El agotamiento de la modernidad (2014), Franco "Bifo" Berardi, ensaya en torno a la percepción psicológica que emergió en el contexto cultural de la modernidad progresiva, así como las expectativas culturales que fueron construidas a lo largo de lo que la historiografía tradicional consigna como civilización moderna, las cuales, en la propuesta de Berardi, alcanzaron su apogeo luego de la Segunda Guerra Mundial.

Según el filósofo italiano, tales expectativas se fueron imponiendo mediante formaciones discursivas cuyo marco conceptual presentaba un desarrollo siempre progresivo, aunque por medio de diferentes construcciones metodológicas: "la mitología de la Aufhebung hegeliano-marxista y la fundación de la nueva totalidad del comunismo; la mitología burguesa de un desarrollo lineal del bienestar y la democracia; la mitología tecnocrática del poder universal del conocimiento científico; y así sucesivamente". A su vez, para Mark Fisher en Los fantasmas de mi vida. Escritos sobre depresión, hauntología y futuros perdidos (2018), una de las consecuencias que puede percibirse contemporáneamente con respecto a esta experiencia retrospectiva o anacrónica con la temporalidad cultural ha sido que, a pesar de "toda la retórica de la novedad y la innovación, el capitalismo neoliberal ha privado gradual pero sistemáticamente a los artistas de los recursos necesarios para producir lo nuevo" (40). En un planteamiento en el que resuena el diálogo sostenido con Berardi y Reynolds, además de Fredric Jameson y su señero estudio La lógica cultural del capitalismo tardío, Fisher señala que mientras "la cultura experimental del siglo xx estuvo dominada por un delirio recombinatorio que nos hizo sentir que la novedad estaría disponible infinitamente, el siglo xxı se ve oprimido por una aplastante sensación de finitud y agotamiento" (32).

Colocado en perspectiva histórica, no resulta difícil advertir que esta complejidad para "producir lo nuevo" en la que se detiene Mark Fisher - y también Simon Reynolds en Retromanía (2012) - aparece como una experiencia significativa dentro del arco de la modernidad, sobre todo aquel trecho que puede recortarse desde las medianías del siglo xix en adelante. Esto debido a que podemos pensar que, en el plano de los discursos críticos sobre la cultura, quizás el punto más condensado para reconocer los cambios que involucran a este tipo de prácticas, sobre todo artísticas, sea a partir de las valorizaciones, y desvaloraciones, por cierto, que largamente recibieron durante el periodo consignado como modernismo, las atenciones a lo insólito, lo inaudito, lo inusitado. Es decir, aquello que el campo de lo inteligible irrumpe dando la impresión de presentarse como "algo nuevo". Podemos encontrar en "Parque central" de Walter Benjamin una rápida tipología distintiva de posiciones generales adoptadas y defendidas ante aquellos objetos que fueron percibidos ya sea como novedad o como "eterno retorno". Figura paradigmática que Benjamin vio particularmente en Charles Baudelaire, de quien llegó a escribir que la poesía del francés "hace manifiesto lo que es nuevo en lo que es siempre-de-nuevo-igual, así como lo siempre-de-nuevo-igual, a su vez, en lo nuevo" (280). No obstante, esta posición paradigmática de Baudelai- 
re encuentra, para Benjamin, figuras opositoras en Friedrich Nietzsche o Auguste Blanqui, a partir de la toma de posicionamientos distintivos que recorren una dialéctica dinamizada por "lo nuevo" enfrentado a "lo siempre de nuevo igual". Puede leerse lo siguiente en un apunte integrante de "Parque central":

Presentar en detalle el hecho de que la idea del eterno retorno penetra más o menos simultáneamente en el mundo de Baudelaire, de Blanqui y de Nietzsche. En Baudelaire el acento recae sobre lo nuevo que, con esfuerzo heroico, se arranca a lo "siempre-de-nuevo-igual", en Nietzsche en cambio en lo "siempre-de-nuevoigual” que el hombre afronta con heroica compostura. Blanqui está más cerca por su parte respecto a Nietzsche que a Baudelaire, pero en él predomina la resignación. En Nietzsche esta experiencia se proyecta cosmológicamente en esta tesis: ya nada nuevo ocurre (280).

Por tanto, esta pugna por percibir ya sea positiva, resignada, escéptica o antagónicamente "lo nuevo", puede verse como la estrategia que ha actuado de modo más general en la configuración y dinamización de campos culturales durante gran parte de la modernidad. Estrategias que, por cierto, también han sido leídas como uno de los modos en que los sujetos intentaron autorizarse y asimismo desautorizar posiciones agonísticas en la conformación de las “arenas culturales", utilizando la célebre expresión acuñada por Richard Morse, las que, a su vez, se vieron modificadas a través de las sucesivas transformaciones impulsadas por el capitalismo de consumo. A propósito del hito que actúa como referencia inicial del tramo histórico recortado anteriormente - mediados del siglo XIX - vale traer a mención una intensa frase que Karl Marx apuntó en sus Manuscritos económico-filosóficos de 1844, redactados entre marzo y septiembre de aquel año: "La educación de los cinco sentidos es el trabajo completo de la historia universal hasta ahora". Pues resulta significativo advertir que casi en paralelo a este momento histórico, estudios contemporáneos sobre la percepción hayan convenido trazar una transformación radical de nuestros aparatos sensoriales. En efecto, en términos perceptivos, la modernidad ha sido definida como una continua reconfiguración de las capacidades de quienes observan ante la sobreabundancia de formas de consumo sensorial. De acuerdo a Jonathan Crary, si el capital es entendido como "un proceso de aceleración del intercambio y la circulación", puede seguirse que uno de sus efectos inevitables ha sido, entonces, un trabajo constante de un "tipo de adaptabilidad perceptiva humana convirtiéndose en un régimen de atención y distracción recíprocas" (38). De este modo, uno de los aspectos cruciales de la modernidad, en opinión de Crary, es una crisis continua de la capacidad de atención, en la que "las configuraciones cambiantes del capitalismo continuamente fuerzan la atención y la distracción al límite, con una secuencia inacabable de nuevos productos, fuentes de estímulo y flujos de información, para después responder con nuevos métodos de dirigir y regular la percepción" (23). La dialéctica benjaminiana entre "lo nuevo" en relación a "lo siempre-de-nuevo-igual" puede ser explicada a 
partir de este planteamiento en torno a la continua capacidad de atención exigida por - y característica de - la modernidad. Jonathan Crary asevera que a diferencia de lo que ocurría en modelos de visión anteriores a los que emergen a mediados del siglo XIX, "la movilidad, la novedad y la distracción se identificaban como elementos constitutivos de la experiencia perceptiva" (38).

Si mediados de siglo xix puede recortarse como hito de inicio, podemos ubicar una referencia de cierre hacia el decenio de 1980 si seguimos una ilustración proporcionada por Fisher en Los fantasmas de mi vida. En determinado momento de su argumentación el crítico cultural británico sugiere que en 1981 "la década de 1960 parecía mucho más lejana de lo que parece hoy" (32). Para tal efecto Fisher propone el ejercicio de contar "veinticinco años hacia atrás a partir de 1980 y se encontrarán en los comienzos del rock and roll. En 1980, un disco con el sonido de Buddy Holly o Elvis habría parecido extemporáneo [...] Si los Artic Monkeys no se posicionaron como un grupo 'retro' fue parcialmente porque no había un 'ahora' con el que contrastar su retrospección" (32). Es básicamente este fenómeno a lo que Fisher denomina en su propuesta como "posmodernismo retro" o "anacronismo posmoderno", y que en la cultura de masas pueden percibirse de manera más notoria en los recurrentes retornos a giros estéticos identificados con la década de los ochenta en, por ejemplo, series de televisión o películas, o a corrientes identificadas con los noventa, en el ámbito musical. A este fenómeno subyacen como condición los cambios introducidos por el neoliberalismo en torno al vínculo entre trabajo y ocio, entre el tiempo del capital y el tiempo social disponible para salirse del trabajo. Fisher señala que en la "medida en que el servicio público se mercantilizó, surgió una tendencia creciente a distribuir productos culturales que se parecían a lo que ya era exitoso" (41). Contribuyendo esto último a instalar un punto de quiebre en la valorización de "la novedad".

A partir de este encuadre, en el que se pone de relieve el estatus que "lo nuevo" sostuvo durante un larguísimo periodo de tiempo de lo que hemos denominado como modernidad, quisiera presentar una reflexión comparada en torno a la irrupción en sus respectivos campos culturales de tres escritoras que, precisamente, generaron estrategias de legitimación en la década del sesenta mediante la valorización de objetos percibidos y resaltados como portadores de algo nuevo. Estas escritoras son la norteamericana Susan Sontag, la argentino-colombiana Marta Traba y la brasileña Leyla Perrone-Moisés, las cuales además coincidieron en algunos de los objetos en que centraron su atención para irrumpir en sus escenas culturales: el "noveau roman" o el pensamiento de Roland Barthes que vincula particularmente a Perrone-Moisés con Sontag o la pintura figurativa que enlaza las atenciones de las tres (de hecho, Leyla Perrone-Moisés se inicia como pintora atraída por este estilo²), son solo dos ejemplos

2 Un relato autobiográfico sobre este punto puede leerse en la entrevista que Jorge Wolff realiza a Leyla PerroneMoisés e incluye en su libro Telquelismos latinoamericanos. La teoría crítica francesa en el entre-lugar de los trópicos (Buenos Aires, Grumo, 2009). 
notorios que podemos mencionar rápidamente. Además, se adicionan al menos dos vectores al problema de legitimación a partir de lo novedoso en el caso de estas intelectuales, si pensamos que se le adhiere a la marca de género otra marcación que las presenta como sujetos debutantes en sus respectivos campos culturales.

Una serie de rasgos en común permiten pensar de manera relacionada ciertas zonas de las obras desarrolladas por Susan Sontag, Marta Traba y Leyla Perrone-Moisés. Por una parte, fueron prácticamente contemporáneas; tan solo seis años de diferencia median entre el nacimiento de la mayor del grupo (Marta Traba, 1930-1983) y la menor (Leyla Perrone-Moisés, 1936). Por otra, las tres se destacaron en el terreno del ensayo, siendo algunos de sus objetos de atención privilegiados la literatura y el arte (Traba y Sontag, además, publicaron varias novelas y libros de relatos). También, en las tres escritoras, la década del sesenta fue un periodo importante en sus respectivas bibliografías. En esa década, o entregaron a la imprenta sus primeros libros (casos de Sontag y de Perrone-Moisés), o publicaron algunos de sus textos fundamentales (como en el caso de Traba). Por último, pero no por ello un hecho menor, sino que al contrario, relevante para el presente trabajo, una preocupación compartida subyace a los libros de ensayos que tanto Sontag como Traba y Perrone-Moisés publicaron en la década antedicha: su reflexión sobre la categoría de estilo. Si de modo general su valorización y defensa de objetos culturales percibidos como "novedosos" marca la entrada agonística que tales escritoras llevan a cabo en sus respectivos contextos de producción, ${ }^{3}$ conceptualmente dicha posición construida encuentra un punto de reflexión destacado en la escurridiza y proteica noción de estilo. En lo que sigue, este trabajo intentará pensar de manera comparada y contrastiva los puntos de contacto y de diferencia que sobre el concepto de estilo expusieron Susan Sontag en su clásico ensayo "Sobre el estilo" (incluido en su célebre libro Contra la interpretación y otros ensayos, 1966), Marta Traba en su importante texto Los cuatro monstruos cardinales (1965) - el cual si bien no es su primer libro es, sin duda, un hito importante en el inicio de su producción intelectual- y Leyla Perrone-Moisés en su primer libro $O$ nôvo romance francês (1966), en el que reunió algunas de las columnas sobre literatura francesa que había comenzado a publicar desde inicios de la década de los sesenta en el importante Suplemento Literario de O Estado de São Paulo.

3 Para una profundización de los contextos en que escribieron las tres ensayistas comentadas en este artículo pueden revisarse los siguientes textos. Para el caso de Sontag: Hardwick, Elizabeth. A Susan Sontag Reader. Londres, Penguin, 1982; Pogue, Leland (ed.) Conversations with Susan Sontag. Jackson, University Press of Mississipi, 1995; Schreiber, Daniel. Susan Sontag. Intelectualidad y glamour. Una biografía. Santiago, Tajamar, 2016. Para Marta Traba pueden consultarse los libros de Verlichak, Victoria. Marta Traba: una terquedad furibunda. Buenos Aires, Universidad Tres de Febrero-Fundación Proa, 2001; Gómez Echeverri, Nicolás. En blanco y negro, Marta Traba en la televisión colombiana, 1954-1958. Bogotá, Universidad de los Andes, 2008; Giraldo, Efrén. Marta Traba: crítica del arte latinoamericano. Medellín, La carreta editores, 2007. Por último, para el caso de Leyla Perrone-Moisés, puede revisarse el libro de Jorge Wolff citado en la nota anterior, además de la tesis doctoral de Brandini, Laura Taddei. Imagens de Roland Barthes no Brasil. São Paulo, Universidade de São Paulo, Faculdade de Filosofia, Letras e Ciências Humanas, 2013. 


\section{Sobre el estilo. Funciones de la crítica}

En relación con lo desarrollado anteriormente podemos parafrasear el planteamiento de Benjamin con respecto a Baudelaire y señalar que la estrategia argumentativa que atraviesa de modo más general Contra la interpretación y otros ensayos de Susan Sontag es desvelar "lo que es nuevo en lo que es siempre-de-nuevo-igual, así como lo siempre-de-nuevo-igual, a su vez, en lo nuevo". Esto lo podemos observar tanto en los objetos de atención que se aglutinan en el libro (como el "noveau roman” francés, la tardía y parcelada recepción de György Lukács en Norteamérica, el pensamiento contracultural de Norman O. Brown o el cine de Bresson, Godard, Resnais o Jack Smith, por mencionar algunos ejemplos) como en su planteamiento más propiamente teórico, el cual sobre todo se condensa en el ensayo que da título al volumen, además de otros como "Sobre el estilo", "Notas sobre lo camp" o "Una cultura y la nueva sensibilidad". Precisamente, y como se citó al inicio del texto, en el epílogo agregado en 1996 a Contra la interpretación y otros ensayos, la ensayista y narradora expresará que aunque "no asumía un compromiso de programa con lo 'moderno', adoptar la causa de la obra nueva sobre todo la que había sido desdeñada o ignorada o incomprendida, parecía más útil que escribir sobre añejas predicciones" (390, cursiva agregada). A partir de esta estrategia argumentativa, Sontag generó las condiciones de irrupción en su respectivo campo cultural y, en este sentido, no deja de percibirse como indicativo de esta operación el asunto de que, en los ensayos recientemente señalados, sea frecuente encontrarse frases aforísticas, enunciados que actúan al modo de máximas (“tono exhortatorio" dirá Sontag en el ya citado epílogo a su libro agregado en 1996).

Este planteamiento más amplio y teórico adoptado por Sontag (y que como veremos también puede ser extensible a los casos de Marta Traba y Leyla Perrone-Moisés) podemos, a su vez, reducirlo en sus puntos nodales a lo siguiente. Para la Susan Sontag que pensó esa serie de valores que le interesaron y que se encontraban circulando en la década de los sesenta, la cultura en tanto fenómeno estaba ineluctablemente basada en el exceso, en la superproducción, de manera similar a lo citado anteriormente de Crary, no obstante su resultado por lo general se veía expresado en "la constante declinación de la agudeza de nuestra experiencia sensorial”. Declaró Sontag en su ensayo "Contra la interpretación" que "las condiciones de la vida moderna — su abundancia material, su exagerado abigarramiento- se conjugan para embotar nuestras facultades sensoriales". Ante este diagnóstico global, podemos apreciar el surgimiento de una discrepancia percibida entre "lo nuevo y lo siempre-de-nuevo-igual" y que la ensayista veía asentada largamente en el discurso teórico sobre el arte bajo la figura de una dicotomía tautológica que subyacía a la estructura contenido-interpretación y que generaba una primacía del primero. Para la escritora norteamericana el hecho de que la teoría mimética al imponer al arte el que se defienda a sí mismo "engendra la singular concepción según la cual algo, que hemos aprendido a denominar forma, 
está separado de algo que hemos aprendido a llamar contenido, y la bienintencionada tendencia que considera esencial el contenido y accesoria a la forma" (14).

En esta línea argumentativa, para Sontag la idea de contenido parecía entonces "sobre todo un obstáculo, un fastidio, un sutil, o no tan sutil, filisteísmo" ("Contra" 15). $\mathrm{Y}$ a su vez, uno de los modos recurrentes en lo que se manifestaba la persistencia de esta dualidad guardaba relación con el concepto de estilo enfrentado al de contenido, ya que prácticamente todas las metáforas empleadas para referir al primero de estos dos conceptos acababan por situar la materia en lo interior y el estilo en lo exterior, posicionamiento que Sontag percibía como una "conformidad beata" ("Contra" 29). Dicha ambivalencia alrededor del estilo se encontraba, a juicio de la norteamericana, arraigada en la pasión de toda una cultura, una pasión que llevaba a defender conceptos externos al arte, situados más bien en regímenes de verdad y moralidad. Ambivalencia mal disimulada que incluso podía apreciarse en el examen de la autora en aquellas posiciones que se arrogaban una perspectiva distinta de la noción de estilo como algo externo ("Se creen lo bastante respaldados por un rechazo teórico de la noción vulgar según la cual el estilo trasciende el contenido, mientras sus juicios continúan reforzando precisamente lo que, en el plano teórico, se apresuran a negar"; "Contra” 30). Es decir que Sontag en su planteamiento empujaba a la problemática hacia la frontera de sus fundamentos: "Tras la ambivalencia ante el estilo existe, en último término, la confusión histórica occidental sobre la relación entre arte y moralidad, lo estético y lo ético” (39).

No obstante, para la Sontag que irrumpió con fuerza en la década de los sesenta, una obra de arte "es una cosa en el mundo, y no solo un texto o un comentario sobre el mundo". De allí se derivará su propuesta de que lo que se necesita, "en primer término, es una mayor atención a la forma en el arte. Si la excesiva atención al contenido provoca una arrogancia de la interpretación, la descripción más extensa y concienzuda de la forma la silenciará. Lo que se necesita es un vocabulario, más que prescriptivo, descriptivo, de las formas. La mejor crítica, y no es frecuente, procede a disolver las consideraciones sobre el contenido en consideraciones sobre la forma" ("Contra" 25). Una función de la crítica que atendiera a la forma conllevaba para Sontag el camino hacia una evasión del embotamiento de los sentidos generado por la superabundancia sensorial moderna. "Lo que ahora importa es recuperar nuestros sentidos. Debemos aprender a ver más, a oír más, a sentir más... Nuestra misión consiste en reducir el contenido de modo que podamos ver en detalle el objeto... La función de la crítica debería consistir en mostrar cómo lo que es, incluso que es lo que es, y no en mostrar qué significa" (27). Operación que la autora consignará en la "Necesidad de una erótica del arte" (27).

De momento, quedémonos con la frase "la función de la crítica debería consistir en mostrar cómo lo que es, incluso que es lo que es, y no en mostrar qué significa” para avanzar hacia algunos planteamientos bastante similares acogidos por aquella misma década de los sesenta por la ensayista brasileña Leyla Perrone-Moisés. Un punto de convergencia hacia esta posición fue Roland Barthes, a quien precisamente Susan 
Sontag le dedicó varios escritos, y al que Leyla Perrone-Moisés se abocó a recepcionar y difundir en el ámbito brasileño, dedicándole una reflexión plasmada en artículos y libros, y a la vez traduciendo y prologando varios de los libros del francés. De Barthes, en concreto, la ensayista brasileña adoptará el planteamiento de que el sistema del lenguaje no es análogo al sistema del mundo, sino más bien homólogo. Leyla PerroneMoisés toma de Barthes el concepto de literatura como un "sistema deceptivo", el cual se encuentra caracterizado por la "suspensión del sentido". Podríamos pensar que es este "sistema deceptivo" que apunta a la suspensión del sentido lo que conduce a Leyla Perrone-Moisés a desvelar "lo que es nuevo en lo que es siempre-de-nuevo-igual, así como lo siempre-de-nuevo-igual, a su vez, en lo nuevo". Se pone en juego en el marco de este planteamiento una distinción esencial realizada por Barthes en la década del sesenta y tomada por Perrone-Moisés para leer la literatura en general y en específico para leer el "noveau roman" francés desde Brasil, me refiero a la distinción establecida dentro de la polaridad sentido/significación. Para Barthes, el sentido guardaba relación con el contenido o significado al interior de un sistema significante, mientras que la significación hacía referencia al proceso sistemático que enlaza un sentido y una forma o, en otra opción lexical clave y operativa para la lingüística y el estructuralismo, a un significante con un significado. Aquí la idea de proceso sistemático intenta generar una ruptura con respecto a la comprensión dicotómica de la polaridad. A partir de este marco de relaciones, la literatura para Barthes no debía verse vinculada al sentido, sino que más bien debía comprenderse como el proceso de producción de los sentidos, el proceso productor de la significación. En el prólogo a su traducción de Critique et verité, un texto relevante de la diatriba sostenida por Roland Barthes en el escenario francés de los sesenta para imponerse como actor dentro de tal campo, Leyla Perrone-Moisés asume que "A função da crítica não é pois descobrir e explicar o sentido de uma obra literária, mas descrever o funcionamento do sistema produtor de significação. Não o que a obra significa, mas como a obra chega a significar" (910). ${ }^{4}$ Planteamiento que ya puede percibirse al recorrer las páginas de su primer libro O nôvo romance francês (1966), y en el que inevitablemente resuenan similitudes con la postura teórica defendida por Susan Sontag en aquella misma década y sintetizada en los párrafos anteriores.

Las convergentes tomas de posición teórica sostenidas por Sontag y PerroneMoisés durante la década de los sesenta pueden ser comprendidas como un desplazamiento a nivel ontológico con respecto a las dicotomías tradicionales que sustentaban el discurso sobre el arte. En el epílogo a Contra la interpretación y otros ensayos de 1996, concretamente la norteamericana expresó que las “jerarquías (alto/bajo) y las polaridades (forma/contenido), intelecto/sentimiento) que ponían en entredicho

4 "La función de la crítica no es pues descubrir y explicar el sentido de una obra literaria, sino describir el funcionamiento del sistema productor de significación. No lo que la obra significa sino como la obra llega a significar" (traducción nuestra). 
inhibían la correcta comprensión de la obra nueva que admiraba" (390, cursiva agregada). En la apertura a su libro Los cuatro monstruos cardinales se puede leer una convergencia en torno a esta operación, entre Marta Traba y las dos ensayistas anteriores, convergencia ilustrada a partir del siguiente pasaje en el que la crítica de arte argentino-colombiana relata su particular encuentro con Francis Bacon, Willem de Kooning, José Luis Cuevas y Jean Dubuffet, los cuatro monstruos cardinales:

En un principio hablaba de ellos como de seres ajenos a mí misma, fantásticos y por lo tanto intrasmisibles en su totalidad. No sé en qué momento empecé a convivir con ellos, a ver en la gente que iba por las calles los cuerpos despedazados de De Kooning, las mutilaciones de Cuevas, las geografías de Dubuffet. Los hombres sentados frente a mí en los autobuses quedaban encerrados en las cajas de vidrio de Francis Bacon. Dejé entonces de superponerles ideas, de convertir a los cuadros en torres malabares de mis pensamientos. Ellos fueron cada vez más intransigentes en su relación conmigo. Pasé a pertenecerles y a subyugarme a su tiranía. Creo que esto ocurrió porque eran demasiado vivos para ser formas y porque desde un principio traté de salvarlos de ser solo formas. No estoy arrepentida ni consternada porque se hayan apoderado al fin del libro y anarquizado sus ideas [...] Pero como no se los puede ni afiliar ni clasificar, ni reducir a fórmulas ni macerarlos hasta producir en el mortero experimental un caldo general que suministre o alimente un nuevo lenguaje útil para ser hablado, explicado y seguido por ávidas bandas de divulgadores (11-12, cursiva agregada).

En este pasaje de apertura Traba — al igual que Sontag y Perrone-Moisés — se desentiende de las jerarquías o polaridades estructuradoras de la apreciación en torno a los objetos artísticos y los discursos que se construyen en torno a ellos, se desentiende de la superposición de ideas, para ubicar a tales objetos en una distinta posición ontológica, los que así se apoderaron del libro y anarquizaron sus ideas ("el sistema del lenguaje no es análogo al sistema del mundo, sino más bien homólogo", Perrone-Moisés; "la obra de arte es una cosa en el mundo, y no solo un texto o un comentario sobre el mundo", Sontag). Contra las tipologías, clasificaciones y fórmulas (¿contra la interpretación, al estilo Sontag?), la función de la crítica avanzada por Traba en este pasaje releva la postura de evadir toda dicotomía tautológica, todo saber que se justifica a sí mismo. En torno a esta función de la crítica, tanto Sontag como Perrone-Moisés y Traba se cruzan con una búsqueda. Y esa búsqueda es el estilo.

\section{Devenires del estilo}

Porque en definitiva posicionarse contra la interpretación es ubicarse contra toda taxonomía, tipología o polaridad, y este contra solo puede ser expresado mediante el estilo, si entendemos por tal "el punto en que la vida y la obra se exponen como duelo 
e instauración", al modo en que lo plantea el ensayista y psicoanalista Carlos Kuri en su texto "De la subjetividad del ensayo (problema de género) al sujeto del ensayo (problema de estilo)". Para Kuri, el estilo es un combate con la vida, es el "punto en que la vida no puede pasar al escrito" (190), al menos no de manera programada. Para el ensayista argentino:

\begin{abstract}
El asunto del estilo no debiéramos entenderlo únicamente como la elección que debe hacer todo texto entre cierto número de disponibilidades contenidas en la lengua, cosa que, por otro camino, vuelve a comprimir las cosas en la cuestión del género. Sino, más allá de eso, como la incisión que algunos textos dejan en la lengua; operación que involucra la acción del nombre propio demostrada en la construcción de un lector inédito (194).
\end{abstract}

Estilo como incisión, que nos retrotrae a la raíz etimológica de la palabra, el stylus, aquella punta afilada con la que se hicieron las primeras inscripciones en tablillas. Estilo como incisión en la lengua, el que llega a anarquizar las ideas y se opone a cualquier lenguaje útil para ser hablado en la expresión de Traba, y que hace recordar aquella frase de Gilles Deleuze en la que reflexiona que los devenires "solo pueden ser expresados en un estilo" (8). Para el filósofo francés, un estilo "no es una estructura significante, ni una organización bien pensada, ni una inspiración espontánea, ni una orquestación, ni una musiquilla. Es un agenciamiento, un agenciamiento de enunciación" (8). De allí que quizás el estilo recurrentemente haya sido tratado de definir en una proliferación de sintagmas aseverativos: "el estilo es el hombre" (Buffon), "el estilo es la vida" (Flaubert), "el estilo es la forma de saber lo que se siente y de sentir lo que se sabe" (Unamuno) o incluso la amplia serie de definiciones de estilo que Sontag aglutina en su ensayo sobre el tema. La proliferación aseverativa produce un efecto transgresor y paradójico, imposibilita que una definición se torne taxativa y exclusiva. La proliferación, en definitiva, arroja al estilo a su devenir. Si la paráfrasis es también una forma de incisión, es otra forma de sintagma proliferativo, para concluir podríamos parafrasear aquel aforismo de Sontag que dice "la interpretación es la venganza que se toma el intelecto sobre el arte”, para enunciar que el estilo sería, tal vez, la venganza que se toma la percepción sobre el arte. En sus mejores momentos, los libros inaugurales de Sontag, Traba y Perrone-Moisés aquí comentados abren paso a este reconocimiento.

\title{
Referencias
}

Barthes, Roland. Crítica e verdade. Trad. Leyla Perrone-Moisés. São Paulo, Perspectiva, 2007.

Benjamin, Walter. Obras Libro I/Vol. 2. Madrid, Abada, 2008.

Berardi, Franco. Después del futuro. Desde el futurismo al cyberpunk. El agotamiento de la modernidad. Trad. Giuseppe Maio. Madrid, Enclave de libros, 2014. 
Blanchot, Maurice. La risa de los dioses. Trad. J. A. Doval Liz. Madrid, Taurus, 1976. Crary, Jonathan. Suspensiones de la percepción. Atención, espectáculo y cultura moderna. Trad. Yaiza Hernández Velásquez. Madrid, Akal, 2008.

Deleuze, Gilles. Diálogos. Trad. José Vásquez Pérez. Valencia, Pre-textos, 1980.

Fisher, Mark. Los fantasmas de mi vida. Escritos sobre depresión, hauntología y futuros perdidos. Trad. Fernando Bruno. Buenos Aires, Caja Negra, 2018.

Kuri, Carlos. "De la subjetividad del ensayo (problema de género) al sujeto del ensayo (problema de estilo)". En Giordano, Alberto (ed.). El discurso sobre el ensayo en la cultura argentina desde los 80. Buenos Aires, Santiago Arcos, 2015.

Perrone-Moisés, Leyla. O nôvo romance francês. São Paulo, São Paulo Editora, 1966. - - Roland Barthes: o saber com sabor. São Paulo, Brasiliense, 1983.

Sontag, Susan. Contra la interpretación y otros ensayos. Trad. Horacio Vásquez Rial. Buenos Aires, Debolsillo, 2008.

Traba, Marta. Los cuatro monstruos cardinales. México D. F., Ediciones Era, 1965. 